Draft Version June 6, 2018

Preprint typeset using $\mathrm{L}^{A} \mathrm{~T}_{\mathrm{E}} \mathrm{X}$ style emulateapj v. 11/10/09

\title{
SPECTROPOLARIMETRIC EVIDENCE FOR RADIATIVELY INEFFICIENT ACCRETION IN AN OPTICALLY DULL ACTIVE GALAXY ${ }^{1}$
}

\author{
Jonathan R. Trump, ${ }^{2}$ Tohru Nagao, ${ }^{3}$ Hiro Ikeda,${ }^{3}$ Takashi Murayama, ${ }^{4}$ Christopher D. Impey, ${ }^{5}$ John T. \\ Stocke, ${ }^{6}$ Francesca Civano, ${ }^{7}$ Martin Elvis, ${ }^{7}$ Knud Jahnke, ${ }^{8}$ Brandon C. Kelly, ${ }^{7,9}$ Anton M. Koekemoer, ${ }^{10}$ and \\ YOSHI TANIGUCHI ${ }^{4}$ \\ Draft version June 6, 2018
}

\begin{abstract}
We present Subaru/FOCAS spectropolarimetry of two active galaxies in the Cosmic Evolution Survey. These objects were selected to be optically dull, with the bright X-ray emission of an AGN but missing optical emission lines in our previous spectroscopy. Our new observations show that one target has very weak emission lines consistent with an optically dull AGN, while the other object has strong emission lines typical of a host-diluted Type 2 Seyfert galaxy. In neither source do we observe polarized emission lines, with $3 \sigma$ upper limits of $P_{B L R} \lesssim 2 \%$. This means that the missing broad emission lines (and weaker narrow emission lines) are not due to simple anisotropic obscuration, e.g., by the canonical AGN torus. The weak-lined optically dull AGN exhibits a blue polarized continuum with $P=0.78 \pm 0.07 \%$ at $4400 \AA<\lambda_{\text {rest }}<7200 \AA\left(P=1.37 \pm 0.16 \%\right.$ at $\left.4400 \AA<\lambda_{\text {rest }}<5050 \AA\right)$. The wavelength dependence of this polarized flux is similar to that of an unobscured AGN continuum and represents the intrinsic AGN emission, either as synchrotron emission or the outer part of an accretion disk reflected by a clumpy dust scatterer. Because this intrinsic AGN emission lacks emission lines, this source is likely to have a radiatively inefficient accretion flow.
\end{abstract}

Subject headings: galaxies: active — galaxies: nuclei — quasars: emission lines — accretion, accretion disks

\section{INTRODUCTION}

Deep X-ray surveys have revealed many bright X-ray point sources with weak or no emission lines in their optical spectra (e.g., Elvis et al. 1981; Comastri et al. 2002). Called X-ray bright optically normal galaxies (XBONGs) or "optically dull" AGNs (the name adopted here), these objects require an AGN to produce their high $\mathrm{X}$-ray luminosities but lack the broad or narrow emission line signatures of AGN accretion. Optically dull AGNs make up $\sim 15 \%$ of luminous $\left(L_{X}>10^{42} \mathrm{erg} / \mathrm{s}\right)$ point sources in deep X-ray surveys, and $\sim 25 \%$ of those at $z<$ 1 (Trump et al. 2009a; Trouille et al. 2009; Yan et al. 2010). Three major paradigms exist to explain their Xray brightness and optical dullness: (1) obscuration of both narrow and broad emission lines, (2) dilution by host galaxy starlight, and (3) a physically distinct accretion flow. We explore the evidence for and against each

1 Based on observations with the Subaru Telescope, operated by the National Astronomical Observatory of Japan.

2 University of California Observatories/Lick Observatory, University of California, Santa Cruz, CA 95064

3 Research Center for Space and Cosmic Evolution, Ehime University, 2-5 Bunkyo-cho, Matsuyama 790-8577 Japan

4 Astronomical Institute, Graduate School of Science, Tohoku University, Aramaki, Aoba, Sendai 980-8578 Japan

5 Steward Observatory, University of Arizona, 933 North Cherry Avenue, Tucson, AZ 85721

6 Center for Astrophysics and Space Astronomy, Department of Astrophysical and Planetary Sciences, UCB-389, University of Colorado, Boulder, CO 80309

7 Harvard-Smithsonian Center for Astrophysics, 60 Garden Street, Cambridge, MA 02138

8 Max Planck Institut für Astronomie, Königstuhl 17, D69117 Heidelberg, Germany

9 Hubble Fellow

10 Space Telescope Science Institute, 3700 San Martin Drive, Baltimore, MD 21218 paradigm in turn.

The standard AGN unified model has had great success in using obscuration to explain the differences between optically selected Type 1 (broad-line) and Type 2 (narrow-line) AGNs (Krolik \& Begelman 1988; Antonucci 1993). In the simplest interpretation of this model, all AGNs have the broad optical emission lines and strong UV/optical continua of Type 1 AGNs, but along certain lines of sight these features are obscured within a dusty "torus" a few parsecs from the black hole. In an obscured object the narrow emission lines remain visible because they are excited beyond the obscuring material, and so the lack of a BLR and weaker optical continuum of Type 2 AGNs are attributed to obscuration. Similarly, Comastri et al. (2002) and Civano et al. (2007) suggested that optically dull AGNs have the same physical engine as a Type 1 or Type 2 AGN, but with additional obscuration within a few parsecs completely blocking the ionizing continuum radiation from exciting even the narrow emission lines. Rigby et al. (2006) instead suggest that optically dull AGNs are obscured by extranuclear $(>100 \mathrm{pc})$ gas and dust in the host galaxy, blocking the narrow lines along the observer's line of sight. No matter the physical location of the obscuring material, it must preferentially absorb the optical emission, since optically dull AGNs remain X-ray bright. Indeed, more than half of optically dull AGNs are relatively unobscured $\left(N_{H}<10^{22} \mathrm{~cm}^{-2}\right)$ in the X-rays (Severgnini et al. 2003; Page et al. 2003). Preferentially obscuring the optical emission while remaining X-ray unabsorbed would require extreme gas-to-dust ratios not observed in other AGNs (Maiolino et al. 2001).

Optically dull AGNs could also be Type 2 AGNs with narrow emission lines diluted by a bright host galaxy. 
Indeed, Moran, Filippenko \& Chornock (2002) showed that many $z \sim 0$ AGNs would appear optically dull if observed at $z \sim 1$, since the host galaxy would occupy more of the spectroscopic slit or fiber and consequently overwhelm the AGN emission lines. HST/ACS images additionally show that many optically dull AGN hosts at $z<1$ are edge-on (Rigby et al. 2006) or have a second galaxy falling within the spectroscopic aperture (Trump et al. 2009b). However 10-20\% of local (undiluted) AGNs are optically dull (La Franca et al. 2002; Hornschemeier et al. 2005). And even after removing the host galaxy light by decomposing the spectral energy distribution, $\sim 1 / 3$ of optically dull AGNs have anomalously high X-ray to optical flux ratios (Trump et al. 2009b).

Another possibility is that optically dull AGNs have different accretion physics due to low accretion rates (Yuan \& Narayan 2004). Models have long predicted that an AGN with a low accretion rate $\left(\dot{m} \equiv L_{\text {bol }} / L_{\text {Edd }} \lesssim 0.01\right)$ will have a radiatively inefficient accretion flow (RIAF) within some truncation radius $R_{t}$, with $R_{t}$ defined as where the collisional cooling time is comparable to the accretion time (Begelman, Blandford \& Rees 1984; Narayan et al. 1995). Beyond $R_{t}$, accretion will remain in a standard geometrically thin and optically thick disk with a thermal blackbody spectrum (e.g., Shakura \& Sunvaev 1973). However within $R_{t}$ there are too few collisions to couple the ions and electrons and the gas becomes a two-temperature plasma. The electrons are cooled by bremsstrahlung, synchrotron, and Compton up-scattering, while the ions remain at the virial temperature. This means the flow is geometrically thick and optically thin. A RIAF then lacks much of the optical/UV blackbody emission of an optically thick accretion disk, and consequently cannot ionize and/or excite the broad or narrow optical emission lines seen in other AGNs (Yuan \& Narayan 2004; Trump et al. 2011). Because the ions in the RIAF are only marginally bound, such AGNs should also have strong outflows and be consequently radio luminous. Optically dull AGNs, especially those with high X-ray to optical flux ratios, are indeed observed to be have higher ratios of radio to optical/UV luminosity than Type 1 AGNs (Trump et al. 2009b, 2011). Much of the optical and infrared light in RIAF AGNs may be synchrotron radiation associated with the radio jet $(\mathrm{Ho} 2009)$.

Each of these three paradigms has a different signature in spectropolarimetry. Anisotropic obscuration of the narrow emission lines would leave telltale reflected emission lines in the polarized spectrum (Nagao et al. 2004). On the other hand, if an optically dull AGN is a Type 2 AGN diluted by a host galaxy, its polarized flux would be equally diluted. (A diluted optically dull AGN might exhibit polarized broad emission lines like those seen in Type 2 AGNs, but the host galaxy would probably overwhelm them to a non-detectable level.) And if optically dull AGNs have RIAFs we might observe a featureless polarized continuum, either from the synchrotron emission associated with the stronger radio jet (e.g., Jannuzi et al. 1994; Cohen et al. 1999) or because we are viewing the naked, lineless continuum (from the disk beyond the RIAF) reflected by a scattering surface in the host galaxy (Antonucci \& Miller 1985; Ogle et al. 1999; Kishimoto et al. 2001). We summarize the polar-
TABLE 1

Paradigms to Describe Optically Dull AGNs

\begin{tabular}{ll}
\hline \hline \multicolumn{1}{c}{ Paradigm } & \multicolumn{1}{c}{ Spectropolarimetry Signature } \\
\hline Obscuration & Polarized emission lines \\
Dilution & No detectable polarization (diluted by starlight) \\
RIAF & Polarized continuum but no polarized emission lines \\
& \\
\hline
\end{tabular}

ization signatures of each paradigm in Table 1 .

\section{TARGETS AND OBSERVATIONS}

We used Subaru/FOCAS to observe the two brightest optically dull AGNs of Trump et al. (2009b), $095849+013220$ and $100036+024929$. The objects have multiwavelength observations from the Cosmic Evolution Survey (COSMOS, Scoville et al. 2007), a survey based on a $1.7 \mathrm{deg}^{2} \mathrm{HST} / \mathrm{ACS}$ treasury program (Koekemoer et al. 2007). Both targets have identifications and redshifts from the XMM-COSMOS AGN spectroscopic campaign with Magellan/IMACS (Trump et al. 2009a). Despite their absorption line optical spectra, they are confirmed as bona-fide AGN using their XMM data (Cappelluti et al. 2009; Brusa et al. 2010): each has an X-ray luminosity of $L_{0.5-10 \mathrm{keV}}>$ $3 \times 10^{42} \mathrm{erg} / \mathrm{s}$, requiring an AGN (Hornschemeier et al. 2001). We show properties of $095849+013220$ and $100036+024929$ in Table 2 .

Trump et al. (2009b) used AGN plus galaxy fits to the optical photometry to suggest that both of these targets are roughly $45 \%$ AGN and $55 \%$ host galaxy in the $i$ band. Table 2 also gives the X-ray to optical flux ratios, given by $X / O=\log f_{X} / f_{O}=\log \left(f_{0.5-2 \mathrm{keV}}\right)+i_{\mathrm{AB}} / 2.5+5.352$. The second target, $100036+024929$, is a good candidate to be a normal AGN diluted by a host galaxy because of its $X / O(\mathrm{AGN})=-0.6$, fitting nicely in the traditional "X-ray AGN locus" of $-1<X / O<$ 1 (Maccacaro et al. 1988). However the first target, $095849+013220$, is anomalously X-ray bright and optically dull, with $X / O=1.1$ and $X / O(\mathrm{AGN})=1.4$. Since starlight dilution would increase the optical emission and decrease the $X / O$ ratio, the high $X / O$ ratio for the first target means it cannot be explained by starlight dilution. Instead $095849+013220$ is a good candidate to host a RIAF (Trump et al. 2009b).

Spectropolarimetric observations of both targets were undertaken using the Faint Object Camera and Spectrograph (FOCAS, Kashikawa et al. 2002) on the $8.2-\mathrm{m}$ Subaru telescope. Each target was observed for 6 hours of total integration in a $0 . \prime 8$-wide center slit. We used the R58 filter and the $150 \mathrm{l} / \mathrm{mm}$ grism, resulting in an observed spectral range of $5800-10000 \AA$ with $\sim 2.8 \AA /$ pixel resolution. During spectropolarimetry observations on FOCAS, a crystal quartz Wallaston prism splits the incident light into ordinary and extraordinary components, which then pass through a rotating achromatic half-wave plate before being recorded simultaneously on the CCD. Instrumental polarization of Subaru/FOCAS using the center slit is negligible $(<0.05 \%)$, as confirmed by our unpolarized standard star exposures. The 6 -hr observations were divided into 9 sets of 10-min exposures at each of 4 half-wave plate position angles $0^{\circ}, 45^{\circ}, 22.5,67^{\circ} .5$.

We used the unpolarized standard star G191B2B to flux calibrate and remove telluric absorption lines, 
TABLE 2

AGN Target Properties

\begin{tabular}{ccccccc}
\hline \hline RA+Dec & $\mathrm{z}$ & $i_{\mathrm{AB}}$ & $\log \left(L_{X}\right)$ & $f_{\mathrm{AGN}^{\mathrm{a}}}$ & $X / O^{\mathrm{b}}$ & $X / O(\mathrm{AGN})^{\mathrm{c}}$ \\
\hline $095849.02+013219.8$ & 0.361 & 18.94 & 44.83 & 0.45 & 1.1 & 1.4 \\
$100036.21+024928.9$ & $0.308^{\mathrm{d}}$ & 18.77 & 43.03 & 0.45 & -1.0 & -0.6 \\
$145658.70+221846.3^{\mathrm{e}}$ & 0.258 & 19.59 & 42.18 & 0.20 & -0.9 & -0.2
\end{tabular}

${ }^{a}$ Fraction of $i$-band emission resulting from the AGN, as estimated from the template fitting of Trump et al. (2009b).

b $X / O=\log f_{X} / f_{O}=\log \left(f_{0.5-2} \mathrm{keV}\right)+i_{\mathrm{AB}} / 2.5+5.352$

${ }^{\mathrm{c}}$ Ratio of X-ray to optical emission considering only the AGN component, including all of the X-ray emission and only $f_{\mathrm{AGN}}$ of the $i$-band emission.

d Note that Trump et al. (2009a) and Trump et al. (2009b) incorrectly reported the redshift for $100036+024929$ as $z=0.47$ with $z_{\text {conf }}=3(\sim 90 \%$ confidence $)$ because they used a Magellan/IMACS spectrum in which $\mathrm{H} \alpha$ fell in a chip gap. The Subaru/FOCAS spectrum presented here reveals that the correct redshift for this target is $z=0.308$.

e This target is the cluster X-ray source MS1455 X-2, a BL Lac candidate discussed in the Appendix.

and used the polarized standard star HD251204 (Turnshek et al. 1990) to determine the absolute position angle. We reduced individual frames using the FOCAS iraf cookbook, and then calculated the normalized flux difference for each individual frame: $F_{\theta}=\left(f_{\theta}^{o}-\right.$ $\left.f_{\theta}^{e}\right) /\left(f_{\theta}^{o}+f_{\theta}^{e}\right)$. We then combined the normalized flux difference for each angle $\theta$ and calculated the Stokes parameters: $Q=0.5\left(F_{0}-F_{45}\right)$ and $U=0.5\left(F_{22.5}-F_{67.5}\right)$. We additionally computed the Stokes parameters using the method of Miller, Robinson, \& Goodrich (1988), which accounts for calibration differences between the "ordinary" and "extraordinary" light positions, and found no significant differences in the resultant Q or U.

The degree of polarization is given by $P \equiv \sqrt{Q^{2}+U^{2}}$ and the polarization angle by $\theta \equiv 0.5 \arctan (U / Q)$. Errors in $U$ and $Q$ bias the total polarization $P$ in the positive direction, and we correct for this bias using the simulation-derived correction of Patat \& Romaniello (2006). Galactic interstellar polarization is $<0.2 \%$ in the direction of both targets (Heiles 2000) and is therefore disregarded.

\section{RESULTS}

The total flux, degree of polarization, and polarization angle for the RIAF candidate and dilution candidate AGNs are shown in Figures 1 and 2, respectively. We bin the degree of polarization and polarization angle by 20 pixels ( $56 \AA$ in the observed frame) to improve their signal-to-noise.

It is first evident that several narrow emission lines are revealed in the unpolarized Subaru/FOCAS spectra, as compared to our previous Magellan/IMACS spectra with much lower S/N. The RIAF candidate, 095849+013220, may have weak $\mathrm{H} \alpha$ emission, although it is strangely blueshifted from the absorption line redshift. The diluted candidate, $100036+024929$, shows strong $\mathrm{H} \alpha$ and [NII] emission (which was missed in the previous Magellan/IMACS spectrum because of a chip gap) and weak $\mathrm{H} \beta$ and [OIII] emission.

Neither of the polarized fluxes in Figures 1 and 2 show evidence for reflected AGN emission lines like $\mathrm{H} \alpha, \mathrm{H} \beta$, or [OIII]. The NaI D $\lambda 5892$ absorption feature might have increased percent polarization, although it is significant at only $\sim 2 \sigma$ in both spectra. If the increased percent polarization of NaI D is real, it is probably caused by the

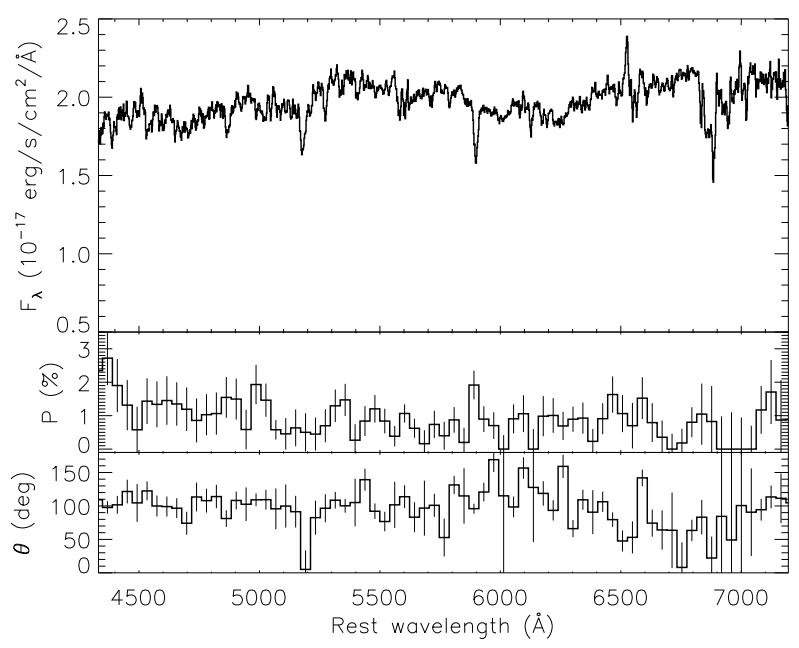

FIG. 1. - Total flux and degree of polarization for 095849+013220, the optically dull AGN likely to have a RIAF. Spectra of polarization degree and angle are binned by 20 pixels ( $41 \AA$ in the rest frame) to improve their $\mathrm{S} / \mathrm{N}$, and the polarization degree is corrected for error bias (Patat \& Romaniello 2006). This target does not show any evidence for polarized AGN emission lines but does exhibit significant continuum polarization, especially in the blue. The overall mean linear polarization is $0.78 \pm 0.07 \%$, and $1.37 \pm 0.16 \%$ at $4400 \AA<\lambda<5050 \AA$, at an angle of $104 \pm 4^{\circ}$.

lower starlight dilution at that wavelength, and so does not represent an actual increase in polarized flux.

The RIAF candidate shows significant continuum polarization, while the diluted candidate does not. The mean continuum polarization of $095849+013220$ is $0.78 \pm$ $0.07 \%$ at an angle of $104 \pm 4 \%$ degrees. The mean continuum polarization in the blue, measured at $4400<$ $\lambda<5050 \AA$, is even stronger: $1.37 \pm 0.16 \%$. Subtracting the host galaxy component, which contributes $\sim 55 \%$ of the total emission at $\lambda_{\text {rest }} \sim 5000 \AA$, suggests that the mean polarization of the AGN component is $\sim 1.7 \%$ overall and $\sim 3.0 \%$ in the blue. The polarized continuum roughly doubles from $\sim 6500 \AA$ to $\sim 4500 \AA$, consistent with a typical unobscured quasar continuum of $f_{\lambda} \sim \lambda^{-1.6}$ (Vanden Berk et al. 2001).

In addition to the two optically dull AGNs from COSMOS, we observed the cluster BL Lac candidate MS 1455-X2 from Hart et al. (2009) and discuss its spectropolarimetry results in the Appendix. 


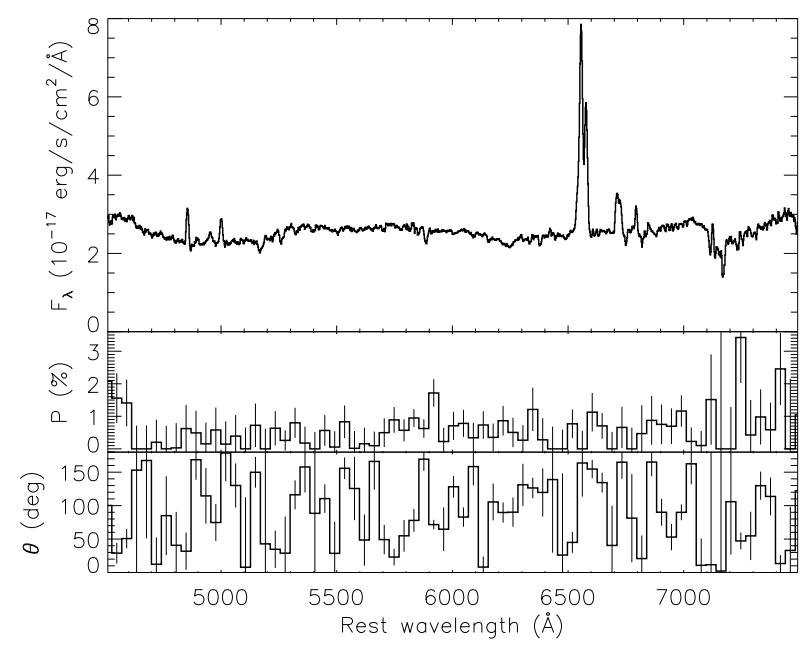

FIG. 2.- Total flux and degree of polarization for $100036+024929$, the optically dull AGN likely to be a Type 2 AGN diluted by its host galaxy. The Subaru/FOCAS spectrum clearly displays $\mathrm{H} \alpha$ and [N $\mathrm{NI}]$ and very weak $\mathrm{H} \beta$ and [O III]. This is in contrast to our previous Magellan/IMACS spectrum, which had much lower $\mathrm{S} / \mathrm{N}$ and showed no emission lines $(\mathrm{H} \alpha$ fell in an IMACS chip gap). As in Figure 1 the polarization degree is corrected for error bias (Patat \& Romaniello 2006) and with the polarization angle is binned by 20 pixels ( $43 \AA$ in the rest frame). This AGN does not exhibit significant linear polarization, either in emission lines or in the continuum.

\section{DISCUSSION}

Neither of our targets show evidence for polarized broad or narrow emission lines, which suggests that the lines are not missing due to anisotropic absorption as in a standard AGN "torus" model (Antonucci 1993). Instead, our RIAF candidate has a blue polarized continuum. Here we place limits on polarized emission line flux and investigate the two possible causes of the continuum polarization: synchrotron emission or scattering.

\subsection{Limits on Polarized Emission Lines}

For both objects the mean polarization in the wavelength regions of $\mathrm{H} \beta$ or $\mathrm{H} \alpha$ broad lines are below or consistent with the measured continuum polarization in the surrounding regions. However a non-detection does not necessarily mean that there are no polarized emission lines, since very weak lines might be undetected within our errors. We approximate each line as a $2000 \mathrm{~km} / \mathrm{s}$ wide top hat, and estimate upper limits on the degree of polarization for $\mathrm{H} \beta$ and $\mathrm{H} \alpha$ broad lines above the polarized continuum using the $3 \sigma$ errors across the line region. For the RIAF candidate $095849+013220$ the $3 \sigma$ upper limits are $2.0 \%$ for $\mathrm{H} \alpha$ and $2.1 \%$ for $\mathrm{H} \beta$, and for the diluted AGN 100036+024929 the $3 \sigma$ upper limits are $1.7 \%$ for $\mathrm{H} \alpha$ and $3.3 \%$ for $\mathrm{H} \beta$. These upper limits decrease for broad lines wider than $2000 \mathrm{~km} / \mathrm{s}$. In contrast, "hiddenBLR" AGNs typically have reflected broad emission lines detected at $P \simeq 5 \%$ (Antonucci 1993; Barth et al. 1999; Moran et al. 2000).

The intrinsic fluxes from both broad lines and continuum are presumably represented in polarized emission. For the RIAF candidate $095849+013220$ we detect a polarized continuum, and can determine upper limits in equivalent width (EW) for broad lines in this intrinsic AGN emission. The $3 \sigma$ upper limits on EW in the intrinsic emission are $\mathrm{EW}_{\mathrm{H} \alpha}<80 \AA$ and $\mathrm{EW}_{\mathrm{H} \beta}<47 \AA$. These limits are less than those of typical quasars, which have $\mathrm{EW}_{\mathrm{H} \beta} \sim 50 \AA$ and $\mathrm{EW}_{\mathrm{H} \alpha} \sim 200 \AA$ (Vanden Berk et al. 2001).

An anisotropic scatterer (e.g., the canonical AGN "torus") is not the reason our two sources lack broad emission lines. Instead both AGNs have weaker broad lines than typical Type 1 or hidden-BLR AGNs, consistent with a RIAF or host galaxy starlight dilution.

\subsection{Continuum Polarization from Synchrotron Emission}

Synchrotron emission from the anisotropic magnetic field associated with a radio jet has a high degree of linear polarization. The RIAF candidate $095849+013220$ could be analogous to BL Lacertae AGNs, which similarly lack both direct and reflected emission lines. BL Lacs typically exhibit linear polarization at $\sim 2-11 \%$, with a median of $\sim 7 \%$ (Jannuzi et al. 1994; Capetti et al. 2007). At first glance the $\sim 1.7 \%$ polarization for the AGN-only component of $095849+013220$ is in the low range of measured polarization for BL Lacs. However the starlight dilution in $095849+013220$ is not well constrained, and the polarization of the AGN component might be significantly higher. Synchrotron emission should extend from radio wavelengths to the optical/UV as a powerlaw, and so the resultant polarized flux should follow the same power-law (e.g., Cohen et al. 1997). The observed increase of polarized flux towards blue wavelengths for $095849+013220$ seems to contradict this, but could again be explained by dilution from an old, red stellar population. The synchrotron polarization of BL Lacs is also strongly variable in amplitude and angle (Jannuzi et al. 1994; Cohen et al. 1997), but we do not have multi-epoch polarization measurements and so cannot perform this test.

Many of the multiwavelength properties of our RIAF candidate are also consistent with those of BL Lacs. The AGN 095849+013220 is radio-luminous (though it sits just below the canonical radio-loudness definition): detected at $F_{1.4 \mathrm{GHz}}=0.646 \mathrm{mJy}$ in the VLA-COSMOS survey (Schinnerer et al. 2010), it has $f_{1.4 \text { Ghz }} / f_{i, A G N}=$ 6.7. This ratio is a factor of a few lower than typical radio to optical ratios for BL Lacs (e.g., Stocke et al. 1990), but again this can be explained if our starlight dilution estimate is too low. X-ray selected BL Lacs (HBLs) have similarly high $X / O$ ratios to $095849+013220$, although the typical $X / O$ of all BL Lacs is lower and more like that of the typical quasar population (Plotkin et al. 2008). The polarized continuum adds another piece of evidence that $095849+013220$ (and possibly other optically dull AGNs with similarly high radio/optical and X-ray/optical ratios from Trump et al. 2009b) might be unified with BL Lacs as another kind of misaligned FR I radio galaxy (Capetti et al. 2007).

\subsection{Continuum Polarization from Scattering}

Scattering also causes continuum polarization, as the observer sees light reflected off the scattering surface. The polarized continuum of $095849+013220$, while blue, actually has the same $f_{\lambda} \sim \nu^{-1.6}$ slope as an unobscured quasar continuum. This slope suggests that optically thick clumpy dust clouds are responsible for the scattering. In contrast the wavelength dependence of op- 
tically thin dust scattering would cause a much bluer slope (e.g. Tran 1995), and electron scattering would require a much larger gas mass than indicated by the Xray column density $\left(N_{H} \simeq 4 \times 10^{20} \mathrm{~cm}^{-2}\right.$, Trump et al. $2009 \mathrm{~b})$. Scattering by clumpy dust is also thought to cause the polarized continuum observed in many Type 1 and Type 2 AGNs (Antonucci \& Miller 1985; Ogle et al. 1999; Kishimoto et al. 2001).

The canonical AGN "torus" is thought to be made of clumpy dust (Nenkova et al. 2008), and so could be the scatterer. The AGN unified model invokes this torus to block and reflect the broad emission lines (Antonucci 1993). In 095849+013220, however, we observe a polarized continuum but no polarized emission lines. Since the broad emission line region is $<<1 \mathrm{pc}$ from the continuum (Elvis 2000; Peterson \& Bentz 2006), it is not physically plausible for any scattering surface to reflect the continuum but not the emission lines. For this reason we interpret the detection of a polarized continuum without broad lines as evidence for a very weak or nonexistent BLR, as expected for radiatively inefficient accretion (Trump et al. 2011).

\section{CONCLUSIONS}

We present Subaru/FOCAS spectropolarimetry for two optically dull AGNs: the RIAF candidate $095849+013220$ and the galaxy-diluted Type 2 AGN candidate $100036+024929$. Neither source exhibits reflected broad emission lines, with $3 \sigma$ upper limits of $\lesssim 2 \%$ polarized broad lines. This rules out simple torus obscuration as the reason behind the lack of broad emission lines. Despite the lack of polarized broad line emission, in the RIAF candidate we additionally observe a polarized continuum. The blue spectral slope of the polarized flux suggests that it represents the intrinsic AGN continuum, either observed directly as synchrotron emission or as reflected by clumpy dust. Because we observe the direct emission from the central AGN engine without broad emission lines, we suggest that this source has an intrinsically weaker or nonexistent BLR. The spectropolarimetry is another piece of evidence that the optically dull AGN 095849+013220 has a radiatively inefficient accretion flow.

We thank Takashi Hattori and the staff of Subaru for excellent support during and after observations. We thank Kenta Matsuoka for help in reducing the FOCAS data. Masaomi Tanaka was invaluable in interpreting the spectropolarimetry results. We also thank Hien Tran and Makoto Kishimoto for help in interpreting a polarized continuum in AGNs. JRT acknowledges support from NSF/DDEP grant \#0943995 and NSF grant \#AST-0808133, and with CDI acknowledges support from \#AST-0908044. FC acknowledges support from the Blancheflor Boncompagni Ludovisi foundation and the Smithsonian Scholarly Studies. BCK acknowledges support from NASA through Hubble Fellowship grant \#HF-51243.01 awarded by the Space Telescope Science Institute, which is operated by the Association of Universities for Research in Astronomy, Inc., for NASA, under contract NAS 5-26555. YT acknowledges support from JSPS grants \#17253001 and \#19340046.

\section{APPENDIX}

\section{OBSERVATIONS OF THE CLUSTER BL LAC CANDIDATE MS 1455-X2}

Hart et al. (2009) reported that the cluster object MS 1455-X2 is luminous in X-ray and radio emission and yet lacks emission lines in its optical spectrum. Its basic properties are given in Table 2. Its optical colors indicate emission blueward of $4000 \AA$ in excess of that expected for a lineless red galaxy, and so MS 1455-X2 is similar to the RIAF candidate we observe in COSMOS. Indeed, Hart et al. (2009) suggest that it is a low-luminosity BL Lac, and predict that such objects may be common in clusters and can provide the feedback necessary to heat cluster cores.

We observed MS 1455-X2 for a total of 160 minutes, divided into 4 sets of 10-min exposures at each of the 4 halfwave plate position angles. We used the same instrumental parameters and reduction process as described in Section 2. The shorter exposure time and faintness of the target $(r=20.05)$ mean that we cannot put meaningful limits on the amount of emission line polarization for MS 1455-X2. However we detected a significant total polarization of $P=2.57 \pm 0.47 \%$ at $4800<\lambda_{\text {rest }}<7700 \AA$. This high degree of polarization strongly supports the hypothesis that this source is a low-luminosity BL Lac AGN, especially considering some amount of starlight dilution from the host galaxy.

\section{REFERENCES}

Antonucci, R. R. J. \& Miller, J. S. 1985, ApJ, 297, 621

Antonucci, R. 1993, ARA\&A, 31, 473

Barth, A. J., Filippenko, A. V. \& Moran, E. C. 1999, ApJ, 525, 673

Begelman, M. C., Blandford, R. D. \& Rees, M. J. 1984, RvMP, 56,255

Brusa, M. et al. 2010, ApJ, 716, 348

Capetti, A. et al.2007, A\&A, 471, 137

Cappelluti, N. et al. 2009, A\&A, 497, 635

Civano, F. et al. 2007, A\&A, 476, 1223

Cohen, M. H., Vermeulen, R. C., Ogle, P. M., Tran, H. D. \& Goodrich, R. W. 1997, ApJ, 484, 193

Cohen, M. H., Ogle, P. M., Tran, H. D., Goodrich, R. W. \& Miller, J. S. 1999, AJ, 118, 1963

Comastri, A. et al. 2002, ApJ, 571, 771
Elvis, M., Schreier, E. J., Tonry, J., Davis, M., \& Huchra, J. P. 1981 ApJ, 246, 20

Elvis, M. 2000, ApJ, 545, 63

Hart, Q. N., Stocke, J. T. \& Hallman, E. J. 2009, ApJ, 705, 854

Heiles, C. 2000, AJ, 119, 923

Ho, L. C. 2009, ApJ, 699, 626

Hornschemeier, A. F. et al. 2001, ApJ, 554, 742

Hornschemeier, A. E., Heckman, T. M., Ptak, A. F., Tremonti, C. A., \& Colbert, E. J. M. 2005, AJ, 129, 86

Jannuzi, B. T., Smith, P. S. \& Elston, R. 1994, ApJ, 428, 130

Kashikawa, N. et al. 2002, PASJ, 54, 819

Kishimoto, M. et al. 2001, ApJ, 547, 667

Koekemoer, A. M. et al. 2007, ApJS, 172, 196

Krolik, J. H., \& Begelman, M. C. 1988, ApJ, 329, 702

La Franca, F. et al. 2002, ApJ, 570, 100 
Maccacaro, T., Gioia, I. M., Wolter, A., Zamorani, G., \& Stocke, J. T. et al. 1988, ApJ, 326, 680

Maiolino, R., Marconi, A., \& Oliva, E. 2001, A\&A, 365, 37

Meier, D. L. 2001, ApJ, 548, L9

Miller, J. S., Robinson, L. B. \& Goodrich, R. W. 1988, in Instrumentation for Ground-Based Optical Astronomy, Present \& Future, ed. L. B. Robinson (New York, NY: Springer-Verlag), 157

Moran, E. C., Barth, A. J, Kay, L. E. \& Filippenko, A. V. 2000, ApJ, 540, L73

Moran, E. C., Filippenko, A. V., \& Chornock, R. 2002, ApJL, 579,71

Nagao, T. et al.2004, AJ, 128, 2066

Narayan, R., Yi, I. \& Mahadevan, R. 1995, Nature, 374, 623

Nenkova, M., Sirocky, M. M., Nikutta, R., Ivezić, Ž. \& Elitzur, M. et al. 2008, ApJ, 685, 160

Ogle, P. M., Cohen, M. H., Miller, J. S., Tran, H. D., Goodrich, R. W. \& Martel, A. R. 1999, ApJS, 125, 1

Page, M. J. et al. 2003, AN, 324, 101

Patat, F. \& Romaniello, M. 2006, PASP, 118, 146

Peterson, B. M. \& Bentz, M. C. 2006, NewAR, 50, 796
Plotkin, R. M. et al.2008, AJ, 135, 2453

Rigby, J. R., Rieke, G. H., Alonso-Herrero, A., \& Perez-Gonzalez, P. G. 2006, ApJ, 645, 115

Schinnerer, E. et al. 2010, ApJS, 188, 384

Scoville, N. et al. 2007, ApJS, 172, 38

Severgnini, P. et al. 2003, A\&A, 406, 483

Shakura, N. I., \& Sunyaev, R. A. 1973, A\&A, 24, 337

Stocke, J. T., Morris, S. L., Gioia, I., Maccacaro, T., Schild, R. E. \& Wolter, A. 1990, ApJ, 348, 141

Tran, H. D. 1995, ApJ, 440, 578

Trouille, L., Barger, A. J., Cowie, L. L., Yang, Y., \& Mushotzky, R. F. 2009, ApJ, 703, 2160

Trump, J. R. et al. 2009a, ApJ, 696, 1195

Trump, J. R. et al. 2009b, ApJ, 706, 797

Trump, J. R. et al. 2011, ApJ submitted

Turnshek, D. A., Bohlin, R. C., Williamson, R. L., II, Lupie, O.

L., Koornneef, J. \& Morgan, D. H. 1990, AJ, 99, 1243

Vanden Berk, D. E. et al. 2001, AJ, 122, 549

Wang, J.-M. \& Zhang, E.-P. 2007, ApJ, 660, 1072

Yan, R. et al. 2010, ApJ submitted (astro-ph/1007.3494)

Yuan, F. \& Narayan, R. 2004, ApJ, 612, 724 